\title{
Pancreatic damage induced by cigarette smoke: The specific pathological effects of cigarette smoke in rat model
}

\section{$\underline{\text { Senay Topsakal }}{ }^{1}$, Ozlem Ozmen $^{2}$, Rahime Aslankoc ${ }^{3}$, Demet Hancer Aydemir ${ }^{4}$}

${ }^{1}$ Department of Endocrinology and Metabolism , Pamukkale University, Faculty of Medicine, Kinikli Campus, Denizli, Turkey. ${ }^{2}$ Department of Pathology, Mehmet Akif Ersoy University, Faculty of Veterinary Medicine, Istiklal Yerleskesi, Burdur, Turkey.

${ }^{3}$ Department of Physiology, Faculty of Medicine, Suleyman Demirel University, Isparta, Turkey.

${ }^{4}$ Department of Medical Laboratory, Health Vocational Schools, Suleyman Demirel University, Isparta, Turkey.

\begin{abstract}
In the modern world smoking is one of the major environmental health risk factor affecting almost all the organs or systems of human body (1). Smoking has become a common serious health and societal problem in last century. It can cause numerous organ and function problems, and occurrence of different diseases including respiratory, cardiovascular, cerebral, and peripheral vascular diseases and especially cancers (2). In relation to pancreatic pathology, smoking has been described as an important risk factor for endocrine and exocrine pancreas functions and the most common environmental risk factor for pancreatic cancer $(1,4)$. Recent human studies reported that the possible increase the risk of pancreatitis in a dose-dependent manner but contradictory some studies reported no correlation with pancreas lesions and smoking (3). It has been reported that smoking increases by approximately 2 -fold the risk of non-gallstone related acute pancreatitis, but not for gallstone-related pancreatitis (4). Andriulli et al. reported that smoking increases $25 \%$ of the risk for the chronic pancreatitis (5). Diabetes mellitus is a group of metabolic diseases characterized by hyperglycemia resulting from defects in insulin secretion or action (6). It is a life-long disease and swiftly increasing in all age groups and both genders. It causes problems in various physiological functions of organs or multiple systems (11), and is associated with wide ranging and devastating health complications (7). The pathogenesis of the damage of smoking on pancreas is, however, not yet well understood, and it remains to be elucidated. Because of these reasons, new experimental studies are needed to explain the effect of cigarette smoke on pancreas. The aim of this study was to examine the pancreas pathology by histopathological, immunohistochemical and biochemical methods in rats exposed cigarette smoking during the 8 weeks and effects of ALA against to cellular damage.
\end{abstract}

Twenty eight female, Spraque Dawley six-months old rats weighing 250-300 g. and cigarette smoke exposures were performed using commercially available filter cigarettes. According to the product specifications, each cigarette contained $1 \mathrm{mg}$ of nicotine, $10 \mathrm{mg}$ of tar and $10 \mathrm{mg}$ carbon monoxide. Animals were inserted into the apparatus to exposed cigarette smoke in successive periods for 1 hour in the morning and 1 hour in the afternoon throughout 7 days a week. Increasing amount of smoke exposure is planned as follows: 1- Sham ( $\mathrm{S})$ group $(\mathrm{n}=8)$; 2- Cigarette smoke exposure (CSE) group $(\mathrm{n}=10)$; Animals were placed into the chamber and exposed to successive periods of cigarette smoke for $1 \mathrm{~h}$ in the morning and $1 \mathrm{~h}$ in the afternoon, 7 days for 8 weeks and given $0.1 \mathrm{ml}$ of physiological serum by orally for 8 weeks simultaneously. 3- CSE+ALA group (n=10); Animals were placed into the chamber and exposed to successive periods of cigarette smoke for $1 \mathrm{~h}$ in the morning and $1 \mathrm{~h}$ in the afternoon, 7 days for 8 weeks and given 100 $\mathrm{mg} / \mathrm{kg} / \mathrm{d}$ orally for 8 weeks; simultaneously. At the end of the experiment, rats were euthanized and after the abdominal incision; blood samples were collected from vena cava inferior and extracted to determine the serum insulin, glucagon, glucose and amylase levels. Pancreases were quickly removed and divided equally into two longitudinal sections. One half of tissues were placed in $10 \%$ neutral formaldehyde solution for routine histopathological and immunohistochemical examinations (caspase-3, CGRP, Hif-1, Hif-2, TNF- $\alpha$, insulin and glucagon). The other half of the tissues were homogenised and kept at $-80^{\circ} \mathrm{C}$ for biochemical studies [Total oxidant status (TOS), total antioxidant status (TAS) levels and oxidative stress index (OSI)]). For histopathological examination; pancreas samples collected during necropsy and fixed $10 \%$ neutral formalin solution. After routine processe, tissues embedded in paraffin and $5 \mu \mathrm{m}$ sectioned. Then sections were stained with hematoxylin- eosin (HE) and examined by light microscope. Selected tissue sections were immunostained by Calcitonin Gene Related Protein, Caspase-3, insulin, glucagon, Hif-1, Hif-2 and TNF antibodies according the manufacturer's instructions.

In this study, statistically significant increase in serum glucose and amylase levels were observed in CSE group and ALA treatment decreased the levels in CSE+ALA group. Results of serum samples were shown in table 1. Serum insulin and glucagon analysis revealed statistically significant decrease in this hormone level but cigarette smoking not significantly affected the serum glucagon level (Table 2). This study results indicated both endocrine and exocrine cell damage due to cigarette smoke exposure in pancreas. In present study cigarette smoke caused statistically increase in TOS and OSI levels ( $\mathrm{p}<0.05$ and $\mathrm{p}<0.05$; respectively), while decrease in TAS levels $(\mathrm{p}<0.001)$. In accordance with these parameters, TOS levels were decreased $(\mathrm{p}<0.05)$ and TAS levels were increased $(\mathrm{p}<0.05)$ in ALA treated groups. Oxidative stress markers of pancreatic tissue were shown in table 2 . These results also supported cigarette smoke induced pancreatic cell damage. Histopathological examination of the pancreases revealed that they generally kept their normal tissue architecture but slight degenerative cells noticed both endocrine and exocrine part of the pancreas in CSE group. Most of the cells exhibited vacuolar and some of the cells exhibited hydropic degeneration (cell swelling). Very rarely cells with pyknotic and karyorrhectic nuclei were also seen. Small number apoptotic cells were observed in pancreas in CSE group. Histopathological evaluation showed no pancreatic inflammation in any group (Fig.1). In S group there were no pathological lesion observed. ALA treatment caused marked amelioration in CSE+ALA groups' pancreatic cells. Immunohistochemistry revealed that decreased insulin secreted cell numbers and severity of expression while slight increase in glucagon secretin cells. In addition immunohistochemically marked increase in active caspase-3, Hif-1, Hif-2, CGRP and TNF- $\alpha$ expressions in both exocrine and Langerhans islets were noticed in CSE group (Figs.2-3). ALA treatment ameliorated the biochemical and pathological findings in CSE+ALA group.

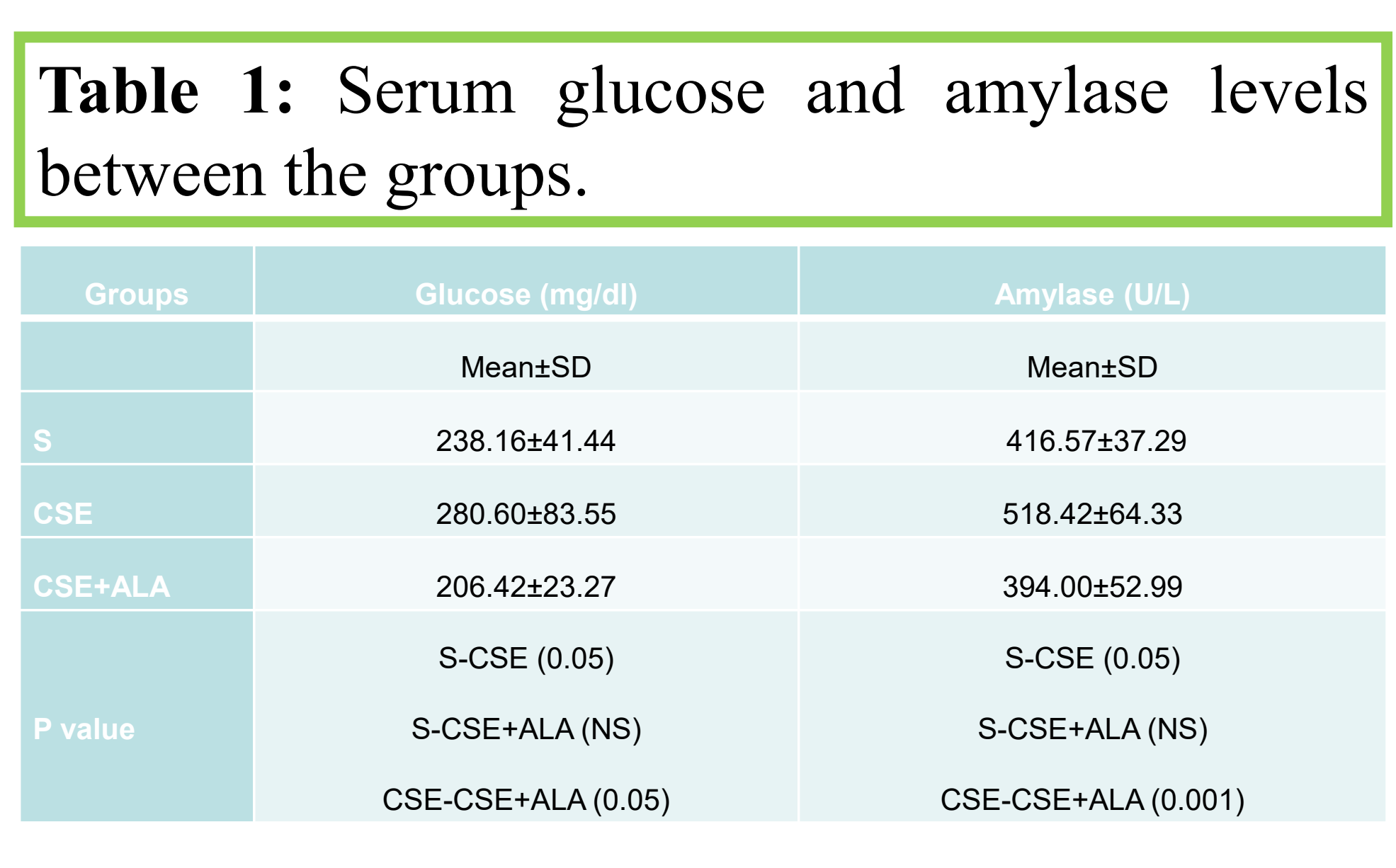

Table 2: Serum insulin and glucagon levels between the groups.

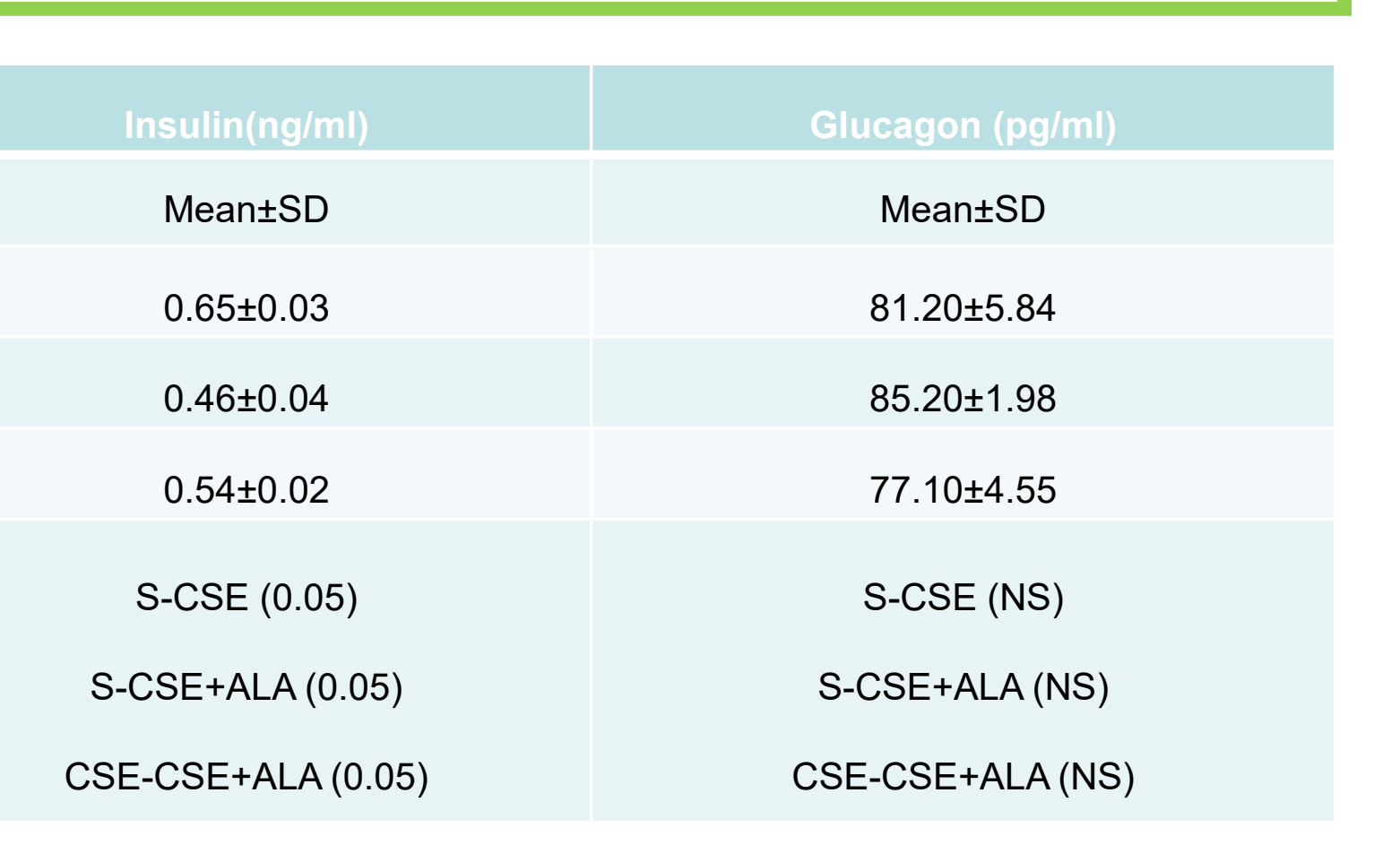

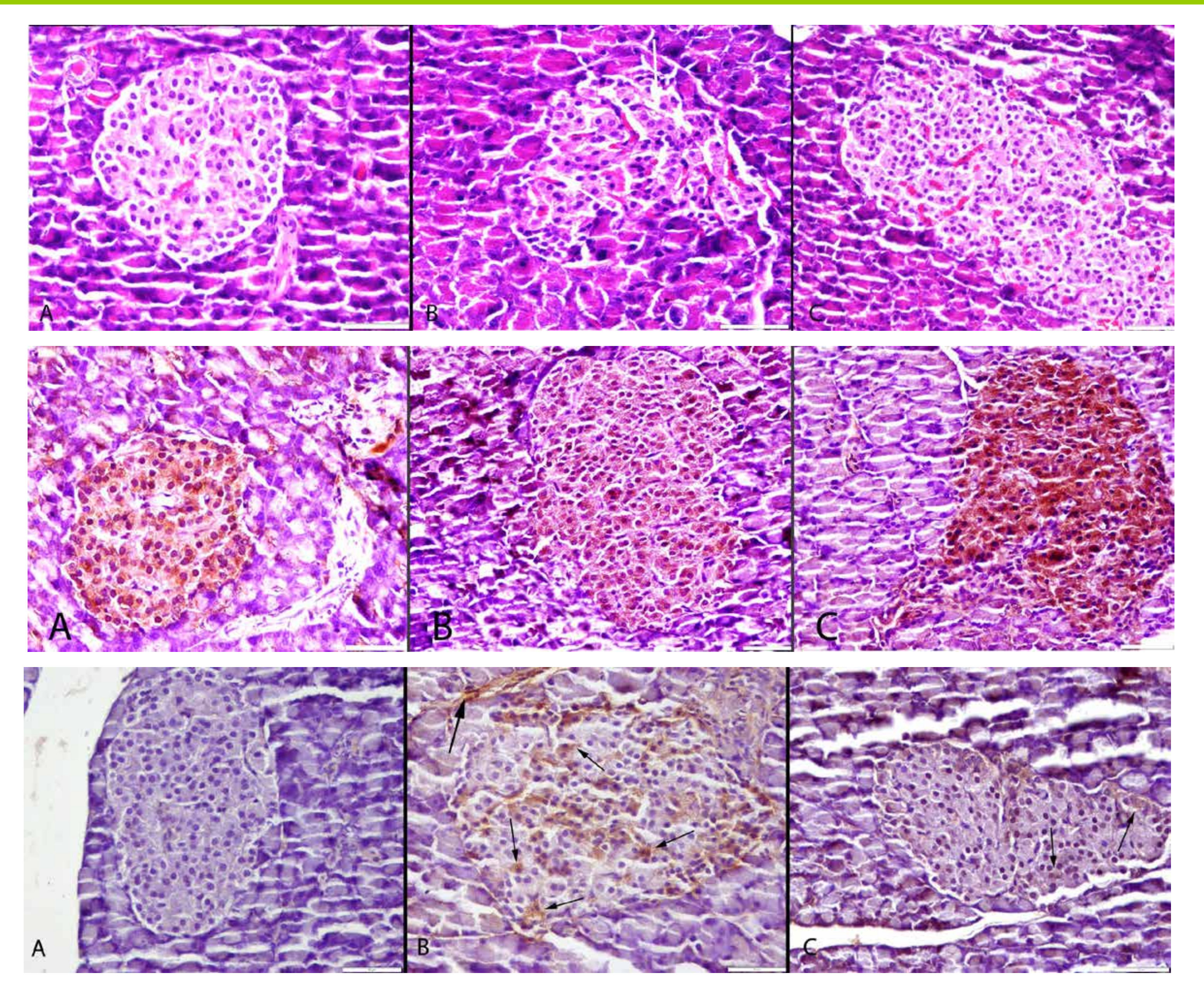

Fig.1: Histopathological appearance of the pancreas. (A) Normal tissue architecture in S group. (B) Slight vacuolar degeneration in both endocrine and exocrine cells (arrows) in CSE group, (C) Normal histology in CSE + ALA group; HE, Bars $=50 \mu \mathrm{m}$.

Fig. 2: Insulin immunoreaction between the groups. Normal expressions in S (A) and CSE+ALA (C) groups. Decrease in severity and insulin secreted cell numbers in Langerhans islet in CSE group (B), Streptavidin Biotin Peroxidase method, Bars= $50 \mu \mathrm{m}$.

Fig.3: Caspase-3 expressions of the groups. (A) Negative caspase-3 immunoreaction in pancreas in S group. (B) Marked increase in endocrine and exocrine cells (arrows) in CSE group. (C) Decreased caspase-3 expression in Langerhans islet cells (arrows) in CSE+ALA group, Streptavidin Biotin Peroxidase method, Bars $=50 \mu \mathrm{m}$. 